\title{
Numerical Evaluation of Self-Preserving Spectra in Smoluchowski's Coagulation Theory
}

\author{
A. MEESTERS* AND M. H. ERNST*, \\ *Institute for Theoretical Physics, State University of Utrecht, P.O. Box 80.006, 3508 TA Utrecht, The Netherlands; \\ and $\dagger$ Institute for Physical Science and Technology, University of Maryland, College Park, Maryland 20742
}

Received October 22, 1986; accepted December 16, 1986

\begin{abstract}
The cluster size distribution $c_{k}(t)$ in aggregation and coagulation phenomena for large cluster sizes $k$ and large times $t$ approaches a scaling form of self-preserving spectrum $c_{k}(t) \sim s^{-2} \varphi(k / s)$, where $s(t)$ is the mean cluster size. In a mean field approach the scaling form $\varphi(x)$ is described by a nonlinear integrodifferential equation, obtained from Smoluchowski's coagulation equation. To verify some theoretical predictions and to provide quantitative information on the scaling form we develop a fixed point method to determine numerical solutions of this equation for aggregation rate constants $K(x, y)=x^{\alpha} y^{\beta}+y^{\alpha} x^{\beta}$ with $\alpha>\beta$ and $\beta<0$ (class III models), where $\varphi(x)$ is bell-shaped, and we study the crossover to class II models $(\beta=0)$, where $\varphi(x) \sim x^{-\tau}$ as $x \downarrow 0$, and calculate the exponent $\tau$. 1987 Academic Press, Inc.
\end{abstract}

\section{INTRODUCTION}

In experiments and in computer simulations on aggregating systems $(1-5)$ the size distribution function for large clusters is seen to approach a universal scaling form when the mean cluster size becomes large. A mean field theory of this aggregation phenomenon is provided by Smoluchowski's coagulation equation, which describes the time evolution of the cluster size distribution $c_{k}(t)$ in a form of an infinite system of coupled nonlinear chemical rate equations for the concentration $c_{k}(t)$ of $k$-mers $(k=1,2, \cdots)$. These findings can be explained from Friedlander's theory of self-preserving spectra (1), which is essentially based on the assumption that, after some initial transient period, the solution of Smoluchowski's equation approaches a similarity or scaling form depending on time and cluster size only through a single argument. Smoluchowski's equation indeed admits similarity solutions, provided the coagulation rates obey certain homogeneity properties $(1,6)$.

There has been a recent revival of interest (4) in Smoluchowski's coagulation equation and, in general, in the kinetics of aggregation processes, instigated by recent computer sim- ulations. There it was found that the resulting clusters have a very tenuous (fractal) structure and one has to reanalyze their geometric and physical properties, such as collision cross sections. Fractal clusters have also been found in many laboratory experiments on gold and silica colloids (4), and soot agglomerates (5).

In these computer simulations (2) one further discovered that the size distribution approached a scaling form for sufficiently large times and sufficiently large cluster sizes. This revived the interest in Smoluchowski's equation, which gave a possible explanation of the above observations.

Before these recent developments, Friedlander's theory was mainly applied to Brownian coagulation in the hydrodynamic and kinetic regimes $(1,11)$ where the rate constants $K_{i j}$ for coagulation of a cluster of size $i$ with one of size $j$ can be calculated from simple hydrodynamic or kinetic theory considerations. In modern applications the mathematical structure of the rate constants is very different from the functional forms used in older applications.

We illustrate this with a clustering process in which the dominant mechanism for cluster 
growth is association of "large with small" (class III process to be defined below), and we consider specifically Brownian coagulation in the kinetic regime where the collision rates $K_{i j}$ $\sim\left(i^{-1}+j^{-1}\right)^{1 / 2}\left(i^{1 / 3}+j^{1 / 3}\right)^{2}$. However in the kinetic collision rates for (fractal) soot agglomerates (6), one must account for the fractal geometry of the cluster with a Hausdorff dimension $D \simeq 1$.9. One can show (15) that the appropriate collision rate for $D \leqslant 2$ becomes now $K_{i j} \sim\left(i^{-1}+j^{-1}\right)^{1 / 2}\left(i^{1 / D}+j^{1 / D}\right)^{D}$. In the size range where both cluster sizes are very different, this $K_{i j}$ is well approximated by $K_{i j} \sim i j^{-1 / 2}+j i^{-1 / 2}$, constituting a special case of the kernel $K_{i j} \sim i^{\alpha} j^{\beta}+j^{\alpha} i^{\beta}$ with $\beta<0$, to be studied numerically in this paper.

A second motivation for the present study derives from an observation, made in analytic studies and computer simulations of clustering processes. If the dominant growth mechanism is not restricted to "large with small" (class III) but depends equally on association of "large with large" clusters (class II), then the scaling function is observed to cross over from a bell-shaped form (class III), as found in Friedlander's theory, to a monotomically decreasing form (class II).

In the latter case, the integral equation for the scaling function, resulting from Friedlander's theory, is ill-defined and must be modified (6). This point has not been noticed in the older literature. There Friedlander's integral equation is, for instance, incorrectly applied to coagulation in shear flow (11), where $K_{i j} \sim\left(i^{1 / 3}+j^{1 / 3}\right)^{3}$ and belongs to class II.

At present no numerical methods seem to exist for determining the scaling function in class II coagulation models. One of the goals of this paper is also to present a numerical limiting procedure for constructing such solutions. The mathematical kernels $K_{i j}=i^{\alpha} j^{\beta}$ $+i^{\beta} j^{\alpha}$ are also used for that purpose.

For later reference we need the theory of self-preserving spectra, yielding a cluster distribution of form

$$
c_{k}(t)=\left(M / s^{2}(t)\right) \varphi(k / s(t)),
$$

where $M=\sum_{k} k c_{k}(t)$ is the total mass or total number of monomers per unit volume and $s(t)$ is some measure for the mean cluster size. The scaling function $\varphi(x)$ is determined by the integro-differential equation

$$
\begin{gathered}
-w\{x d \varphi(x) / d x+2 \varphi(x)\}=\frac{1}{2} \int_{0}^{x} d y K(y, x-y) \\
\times \varphi(y) \varphi(x-y)-\varphi(x) \int_{0}^{\infty} d y K(x, y) \varphi(y) .
\end{gathered}
$$

The kernel $K(x, y)$ is the rate constant for coagulation of an $x$-mer and a $y$-mer, and is supposed to be a homogeneous function of its variables, viz.

$$
K(a x, a y)=a^{\lambda} K(x, y)
$$

with degree of homogeneity $\lambda$. The constant $w$ in Eq. [2] is a (positive) separation constant also entering in the equation of motion for the mean cluster size $s(t)$. For a discussion of the time dependence of $s(t)$ we refer to the literature (6).

The theory has also been extended by van Dongen and Ernst (6) to class I aggregation processes where the dominant growth mechanism is association of large with large clusters, and to the intermediate class II processes, where the rate constants for association of large-large and large-small are of the same order of magnitude. Class I also includes processes that lead to the formation of an infinite cluster or gel within a finite time. More formally, we have

$$
K(x, y) \sim x^{\mu} y^{v} \quad(y \geqslant x),
$$

where $\mu>0, \mu=0$, and $\mu<0$ defines respectively classes I, II, and III. Furthermore, the following physical restrictions have been imposed (6) on the exponents: $\nu \leqslant 1$ and $\lambda=\mu$ $+\nu \leqslant 1$ for nongelling systems and $1<\lambda$ $\leqslant 2$ for gelling systems. The integral equation for the scaling function $\varphi(x)$ in classes I and II is more complicated than Eq. [2] because of canceling infinities in the gain and loss term on the RHS of Eq. [2]. For instance, in non- 
gelling class I and II processes the $\varphi(x)$ equation reads

$$
\begin{aligned}
& -w\{x d \varphi(x) / d x+2 \varphi(x)\} \\
& =\lim _{\epsilon \downarrow 0}\left[\frac{1}{2} \int_{\epsilon x}^{(1-\epsilon) x} d y K(y, x-y) \varphi(y) \varphi(x-y)\right. \\
& \left.\quad-\varphi(x) \int_{\epsilon x}^{\infty} d y K(x, y) \varphi(y)\right] .
\end{aligned}
$$

Analytic solutions to the $\varphi(x)$ equation are only known for three special kernels:

\begin{tabular}{cl}
$K(x, y)$ & $\varphi(x)$ \\
\hline 1 & $e^{-x}$ \\
$x+y$ & $x^{-3 / 2} e^{-x}$ \\
$x y$ & $x^{-5 / 2} e^{-x}$
\end{tabular}

In all other cases only the behavior of the solution $\varphi(x)$ (if such a solution exists) at small $x$ and at large $x$ has been determined analytically. The qualitative behavior of the scaling function differs markedly between class III (where the scaling function has a bell-shaped form and vanishes exponentially fast as $x \downarrow 0$ ) and classes I and II (where the scaling function behaves algebraically: $\varphi \sim x^{-\tau}$ at small $x$ ).

Quantitative information on the scaling function is scarce, as it is in general very difficult to construct an algorithm for numerically solving the integro-differential equations [2] and [5]. This is caused by the loss term which involves $\varphi(x)$ for all values of $x$. The only cases for which numerical solutions have been obtained are class III kernels $K(x, y)$ with factorizable $x$ and $y$ dependence or sums of such kernels.

The coagulation kernels considered in aerosol physics belong to this category, for instance Brownian coagulation with $K(x, y)$ $=2+(x / y)^{1 / 3}+(y / x)^{1 / 3}$. The solution method as well as the numerical values of the "selfpreserving spectrum" $\varphi(x)$ for Brownian coagulation and refinement thereof have been discussed in Friedlander's book (1). More recently Kolb (7) numerically determined the scaling function for the factorizable class III kernel $K(x, y)=(x y)^{\omega}$ with $\omega<0$. He used the last kernel to model diffusion-limited aggregation of clusters.

The main goal of this paper is a numerical study of the solution of the $\varphi(x)$ equation, in order to obtain quantitative data on the scaling form for different types of coagulation processes, and also to test the analytic results of (6) which were mostly derived from self-consistency arguments, and refer only to asymptotic behavior at large or at small $x$. These asymptotic forms contain coefficients, left undetermined by the theory. The relationship between the coefficients, entering in the large$x$ behavior and in the small- $x$ behavior, is also left undetermined. Furthermore, we want to study how crossover occurs from the typical bell-shaped behavior of class III to the algebraic small- $x$ behavior of class II. For the latter class no numerical solutions have been reported so far.

This will be done by studying model kernels of the general type

$K(x, y)=x^{\alpha} y^{\beta}+y^{\alpha} x^{\beta} \quad(\beta \leqslant \alpha \leqslant 1, \beta<0)$

and by letting the class III kernels with $\mu=\beta$ $<0$ approach class II kernels by taking $\beta \uparrow 0$. The plan of the paper is as follows. In Section 2 we review the analytic results obtained for the above class III kernels and for its different limiting forms, all belonging to class II. The numerical solution method is discussed in Section 3 and the results in Section 4. We end with a conclusion.

\section{REVIEW OF ANALYTICAL RESULTS}

It can easily be seen that if some $\varphi$ is a solution of Eq. [2] with a separation constant $w$, then $\bar{\varphi}=\eta^{-1} \varphi(\xi x)$ is a solution of Eq. [2] with $\bar{w}=w \eta^{-1} \xi^{-1-\alpha-\beta}$. In order to fix the constants $\eta$ and $\xi$ we introduce moments $p_{\gamma}$ of the scaling function

$$
p_{\gamma}=\int_{0}^{\infty} d x x^{\gamma} \varphi(x)
$$

One constant is fixed by mass normalization, $\sum k c_{k}=M$, which requires through Eq. [1] that $p_{1}=1$. The second is determined by defining a mean cluster size $s(t)$, for which one 
may take the mass-average degree of polymerization, $s(t)=\sum k^{2} c_{k} / M$ implying $p_{2}=1$, or the number-average degree of polymerization $s(t)=M / \sum c_{k}$ implying $p_{0}=1$ (not allowed for class II--see Eqs. [14] and [16]), or some other relation between the moments. Rescaling of $\varphi(x)$ and $x$ always enables us to transform results obtained for one normalization into a different one. Specific choices of normalization will be needed in later sections.

Existence and uniqueness (apart from scale invariance) have not been proved analytically, but have become highly probable (for classes II and III) by the computation. They will be supposed from now on. We also suppose that the moments $p_{\gamma}$ for class III kernels exist for all $\gamma$.

The $\varphi(x)$ equation for the kernel [6] with $\beta$ $<0$ becomes

$$
\begin{gathered}
-w\{x d \varphi(x) / d x+2 \varphi(x)\}=\frac{1}{2} \int_{0}^{x} d y y^{\alpha}(x-y)^{\beta} \\
\times \varphi(y) \varphi(x-y)-\left(p_{\alpha} x^{\beta}+p_{\beta} x^{\alpha}\right) \varphi(x) .
\end{gathered}
$$

An equivalent representation in terms of Mellin transforms, $p_{\gamma}$, is

$$
\begin{aligned}
& w(\gamma-1) p_{\gamma}=\int_{0}^{\infty} d x \int_{0}^{\infty} d y \\
& \quad \times\left[(x+y)^{\gamma}-x^{\gamma}-y^{\gamma}\right] x^{\alpha} y^{\beta} \varphi(x) \varphi(y)
\end{aligned}
$$

obtained by multiplying [8] by $x^{\gamma}$ and integrating over all $x$. This equation also applies to class II kernels ( $\beta \uparrow 0$ ), provided $\gamma$ is sufficiently large, such that $p_{\gamma}$ exists at the lower limit of integration (see Eqs. [14] and [15] below). In our numerical solutions, Eq. [9] will be used for integer $\gamma$, viz.

$$
\begin{gathered}
p_{0} w=p_{\alpha} p_{\beta}, \\
(n-1) p_{n} w=\sum_{k=1}^{n-1}\left(\begin{array}{l}
n \\
k
\end{array}\right) p_{\alpha+k} p_{\beta+n-k} \\
(n=2,3, \cdots) . \quad[10]
\end{gathered}
$$

Note that only single integrations are required for the evaluation of both sides of this equation.
The large- $x$ behavior of all kernels in [6] with $\alpha<1$ was found in (6)

$$
\begin{array}{rlrl}
\varphi(x) & \simeq A x^{-\alpha-\beta} e^{-a x} & & (\alpha<1), \\
& \simeq B x^{-(3+\beta) / 2} e^{-b x} & (\alpha=1) .
\end{array}
$$

The undetermined constants $A$ and $a$ for $\alpha$ $<1$ are related as

$$
a / A=B(1-\alpha, 1-\beta) / w,
$$

where $B(p, q)=\Gamma(p) \Gamma(q) / \Gamma(p+q)$. The large$x$ behavior for $\alpha=1$ has been deduced from (8), but the relationship between the undetermined constants $B$ and $b$ has not been established.

In classes II and III the small-x behavior of $\varphi(x)$ is very different (6). For class III kernels [6] $(\beta<0)$ the scaling function vanishes exponentially fast as $x \downarrow 0$, viz.

$$
\begin{array}{rlr}
\varphi(x) & \simeq C x^{-2} \exp \left[p_{\alpha} x^{\beta} / \beta w+p_{\beta} x^{\alpha} / \alpha w\right] & \\
& \simeq C x^{-1} \exp \left[p_{0} x^{\beta} / \beta w\right] \quad(\alpha=0), & {[13]}
\end{array}
$$

where $C$ is undetermined.

For the class II kernels, that are the limiting forms of [6] as $\beta \uparrow 0$, viz. $K(x, y)=x^{\alpha}+y^{\alpha}$ (where necessarily $\alpha \geqslant 0$ ), one finds algebraic behavior,

$$
\varphi(x) \simeq B x^{-\tau} \quad(x \downarrow 0),
$$

where $B$ is undetermined and $\tau$ is not known explicitly, but given in terms of moments of $\varphi(x)(9,10)$, viz.

$$
\tau=2-p_{\alpha} / w
$$

We also note that the exponent $\tau$ is independent of the arbitrary scale constants $\eta$ and $\xi$, discussed at the start of Section 2, since the moment $\bar{p}_{\alpha}$ of $\bar{\varphi}$ is related to $p_{\alpha}$ of $\varphi$ as $\bar{p}_{\alpha}$ $=\eta^{-1} \xi^{-1-\alpha} p_{\alpha}$. Hence $\bar{p}_{\alpha} / \bar{w}=p_{\alpha} / w$. The exponent $\tau$ satisfies the inequalities

$$
1<\tau<1+\alpha \quad(\alpha>0),
$$

but better bounds have been derived in (10).

\section{NUMERICAL SOLUTION METHOD}

The numerical method for solving the nonlinear integro-differential equation [8] is largely based on the method used by Swift and 
Friedlander (11) for Brownian coagulation where $K(x, y)=2+(x / y)^{1 / 3}+(y / x)^{1 / 3}$. The feature that enables us to solve the $\varphi(x)$ equation is the simple structure of the loss term containing $\varphi(x)$ multiplied with a function of $x$ with a finite number of unknown coefficients, which are here $p_{\alpha}$ and $p_{\beta}$.

If $\varphi(x)$ is known on the interval $x \in[0, \epsilon]$ for a given pair of values $\left(p_{\alpha}^{(\mathrm{i})}, p_{\beta}^{(\mathrm{i})}\right)$, then $d \varphi(x)$ / $d x$ is known from Eq. [8], and the $\varphi(x)$ equation can be solved by applying Adam's extrapolation method (12) for solving ordinary first-order differential equations. On the starting interval $[0, \epsilon]$ the function can be approximated by its known small- $x$ behavior [13]. Hence, from the pair $\left(p_{\alpha}^{(i)}, p_{\beta}^{(i)}\right)$ follows a $\varphi(x)$, from there we calculate again the pair of moments $\left(p_{\alpha}^{(\mathrm{c})}, p_{\beta}^{(\mathrm{c})}\right)$, so that in principle $\left(p_{\alpha}^{(\mathrm{c})}, p_{\beta}^{(\mathrm{c})}\right)=F\left(p_{\alpha}^{(\mathrm{i})}, p_{\beta}^{(\mathrm{i})}\right)$ for a certain function $F$. The remaining problem is to determine the fixed point of $F$.

To start we recall that the general solution of Eq. [8] contains two arbitrary scale constants $\eta$ and $\xi$ and we observe that the small$x$ behavior in [13], required in our solution method, contains two undetermined constants $C$ and $w$. Thus, a convenient choice of fixing $\eta$ and $\xi$ is by setting $C=1$ and $w=1$.

Next, the function $\varphi(x)$ is transformed into a function $\psi(z)$, which varies more smoothly and is, therefore, better suited for being calculated with constant step size $h$

$$
\begin{aligned}
x & =e^{z} \quad(-\infty<z<\infty), \\
\varphi(x) & =\psi(z) .
\end{aligned}
$$

Hence $\psi$ satisfies

$$
\begin{aligned}
& d \psi(z) / d z=-\int_{0}^{\exp (z)} d y y^{\alpha} \\
& \times\left(e^{z}-y\right)^{\beta} \psi(\ln y) \psi\left(\ln \left(e^{z}-y\right)\right) \\
& \quad+\left[p_{\alpha} e^{\beta z}+p_{\beta} e^{\alpha z}-2\right] \psi(z)
\end{aligned}
$$

The abscissas $z_{j}(j=1,2, \cdots)$ were chosen equidistantly according to the following method: a rough estimate of $\varphi(x)$ for $x$ not too large is given by the asymptotic form [13] with $C=1$ and $w=1$, viz.

Joumal of Colloid and Interface Science, Vol. 119, No. 2, October 1987

$$
\varphi(x) \simeq x^{-\delta} \exp \left[-p x^{-\omega} / \omega\right] \quad(x \downarrow 0)
$$

with $\omega=-\beta>0$ and

$$
\begin{array}{lll}
p=p_{\alpha}, \delta=2 & \text { if } & \alpha>0 \\
p=p_{0}, \delta=1 & \text { if } & \alpha=0 \\
p=2 p_{\alpha}, \delta=2 & \text { if } & \alpha=\beta=-\omega .
\end{array}
$$

This expression yields a priori estimates $z_{\mathrm{b}}$ and $z_{\mathrm{m}}$ for the location of the first inflection point and the maximum of $\psi(z)$, respectively. The step size was chosen to be $h=\left(z_{\mathrm{m}}-z_{\mathrm{b}}\right) / 20$ where a further decrease in $h$ did not change the results. The interval $[0, \epsilon]$, in which the asymptotic form [13] was supposed to be exact, was chosen as $\epsilon=\exp \left(z_{\mathrm{b}}\right)$. Furthermore $z_{\mathrm{b}}$ was identified as $z_{4}$. For the equidistant abscissas $z_{j}(j=1,2,3,4)$ we calculate $\psi_{j}$ and $(d \psi / d z)_{j}$ from [13]. For $x>\epsilon$ or $z_{j}=z_{5}, z_{6}$, .. the function $\psi(z)$ was calculated by solving Eq. [18] using the four-point Adam extrapolation formula. It was observed that decreasing $\epsilon$ did not change the results.

For very large $x$ this method is expected to become inaccurate. The condition

$$
\left|(\psi(z))^{-1} d^{n} \psi(z) / d z^{n}\right| \leqslant h^{-n} \quad(n=1,2,3,4),
$$

required for accuracy of Adam's extrapolation, is no longer satisfied if $\varphi$ behaves according to Eq. [11].

The evaluation method for the gain term in [8], which we conveniently write as

$$
\int_{0}^{x / 2} d y\left[y^{\alpha}(x-y)^{-\omega}+y^{-\omega}(x-y)^{\alpha}\right]
$$

$$
\times \varphi(y) \varphi(x-y)
$$

determines the speed of the whole procedure. It was calculated in the following manner: a new integration variable $q$ was introduced by

$$
\begin{aligned}
q & =y^{-\omega} p / \omega-q_{0}, \\
q_{0} & =(x / 2)^{-\omega} p / \omega
\end{aligned}
$$

so that the integral [20] was transformed into an integral from 0 to $\infty$ of an integrand behaving regularly as $q \downarrow 0$ and as $q^{(\hat{o}-1) / \omega} e^{-q}$ as $q \rightarrow \infty$. The integrand is therefore suited for 
Laguerre quadrature (13) and a 15-point quadrature was chosen.

Once $\varphi$ is calculated, the moments $p_{\gamma}$ can be evaluated by dividing the integral in [7] into three parts:

$$
\begin{aligned}
p_{\gamma}=\int_{0}^{\exp \left(z_{\mathrm{m}}\right)} d x x^{\gamma} \varphi(x) & +\int_{z_{\mathrm{m}}}^{z_{N}} d z e^{(\gamma+1) z} \psi(z) \\
& +\int_{\exp \left(z_{N}\right)}^{\infty} d x x^{\gamma} \varphi(x)
\end{aligned}
$$

where $N$ is the number of abscissas chosen. In the first term we introduced the new integration variable $y$, defined through

$$
x=\left[y \omega / p+\exp \left(-\omega z_{\mathrm{m}}\right)\right]^{-1 / \omega} .
$$

The new integrand is regular at $y=0$ and behaves as $y^{u} e^{-y}$ with $u=(\delta-\gamma-1) /(\omega-1)$ for $y \rightarrow \infty$. The integral was evaluated by $15-$ point Laguerre quadrature.

The second term can give a large contribution when $\gamma$ becomes large. This happens for large- $z$ values where $\varphi\left(e^{z}\right)$ may not yet have reached its asymptotic behavior [11]. This appears to occur for coagulation kernel $K(x, y)$ in [6] with $\alpha>0$ and $\beta$ close to 0 , viz. when $K(x, y)$ approaches $x^{\alpha}+y^{\alpha}$. Here the integration was carried out, simply using equidistant abscissas (100-point Simpson rule).

The third term in [21] is approximated by substituting for $\varphi$ the large- $x$ behavior [11] and using Laguerre quadrature after linear transformation of the integration variable. The undetermined constants $A$ and $a$ in [11] were calculated from the $\varphi(x)$, determined above, by making a least-squares fit of $\ln \left(x^{\alpha+\beta} \varphi(x)\right)$ to $(-a x+\ln A)$ at the highest $x$ values considered.

Finally, the correct (fixed point) values of $p_{\alpha}$ and $p_{\beta}$ were found in the following manner. So far we have only shown how to calculate $\varphi(x)$ for arbitrarily assumed input values, denoted by $\left(p_{\alpha}^{(\mathrm{i})}, p_{\beta}^{(\mathrm{i})}\right)$ and, next, how the moments of this $\varphi(x)$ can be calculated. The calculated values of $p_{\alpha}$ and $p_{\beta}$, obtained in this manner, are denoted by $\left(p_{\alpha}^{(\mathrm{c})}, p_{\beta}^{(\mathrm{c})}\right)$. Thus, we have a function $F:\left(p_{\alpha}^{(\mathrm{i})}, p_{\beta}^{(\mathrm{i})}\right) \rightarrow\left(p_{\alpha}^{(\mathrm{c})}, p_{\beta}^{(\mathrm{c})}\right)$. A fixed point of $F$ could be found by minimizing the function:

$$
f\left(p_{\alpha}^{(\mathrm{i})}, p_{\beta}^{(\mathrm{i})}\right)=\left(1-p_{\alpha}^{(\mathrm{c})} / p_{\alpha}^{(\mathrm{i})}\right)^{2}+\left(1-p_{\beta}^{(\mathrm{c})} / p_{\beta}^{(\mathrm{i})}\right)^{2},
$$

using a quasi-Newton-Raphson technique (14), and amounts to minimizing the relative error in $\left(p_{\alpha}, p_{\beta}\right)$ in the sense of least squares. After adding some refinements to this method the resulting minimum of $f$ was practically always smaller than $10^{-7}$. The computer time needed for this calculation can be considerable, because every evaluation of $f$ involves the calculation of a $\varphi$.

Finally, the number of abscissas $N$ remains to be chosen. It was found by performing the described procedure for finding $p_{\alpha}, p_{\beta}$ for different values of $N$. In each case the resulting $\varphi$ is tested by evaluating both sides of the moment equations [10] with $w=1$ for $n=0,2$, $3, \cdots, 10$. The $N$ value for which the upper bound of the relative difference of both sides of these 10 equations is minimal is taken to be the best value. $N$ depends somewhat on the kernel $K$, and varies only slowly with $\alpha$ and $\beta$.

\section{NUMERICAL RESULTS}

In this section the symbols $\bar{\varphi}(\bar{x})$ and $\bar{p}_{\gamma}$ are used to denote the scaling function and its moments obtained in the numerical computations of the previous section, where the $\varphi$ and $x$ units were chosen through the "numerical normalization," $C=w=1$. The symbols $\varphi(x)$ and $p_{\alpha}$ will denote the same quantities after the rescaling $\varphi(x)=\eta \bar{\varphi}(x / \xi)$. In Subsections 1 and 2 we use the standard normalization, based on the number-average degree of polymerization (see Introduction),

$$
\begin{aligned}
& p_{0}=\int_{0}^{\infty} d x \varphi(x)=1, \\
& p_{1}=\int_{0}^{\infty} d x x \varphi(x)=1,
\end{aligned}
$$

which is also appropriate for the limiting kernel $K(x, y)=2$ as $\omega \downarrow 0$. This can be carried out by choosing $\xi=\bar{p}_{0} / \bar{p}_{1}$ and $\eta=\bar{p}_{1} / \bar{p}_{0}^{2}$. 
We have studied a set of coagulation kernels [6] belonging to class III when the exponent $\beta \uparrow 0$. The limiting kernels belong to class II. In each case special attention has been paid to the transition from class III to class II behavior.

\section{Kernel $1: K(x, y)=2(x y)^{-\omega}$}

As the first example we consider the kernel [6] with $\alpha=\beta=-\omega$, which has also been computed by Kolb (7), using the same normalization [22]. The integral equation [8] contains only one unknown moment $p_{\alpha}=p_{\beta}$ $=p_{-\omega}$. In Fig. 1 the scaling function $\varphi_{\omega}(x)$ for the above kernel is presented in the standard normalization [22]. Also shown is the exact scaling function for the constant kernel ( $\omega$ $=0$ ), which reads in the standard normalization, $\varphi_{0}(x)=e^{-x}$. The scaling function $\varphi_{\omega}(x)$ is seen to approach $\varphi_{0}(x)$ as $\omega \downarrow 0$.

The bell-shaped curves $\varphi_{\omega}(x)$ are typical for class III behavior. The one with the largest maximum corresponds to $\omega=0.95$. As $\omega$ decreases the maximum starts to decrease and to shift to the left. Around $\omega \simeq 0.2$ one sees typical crossover behavior from class III to

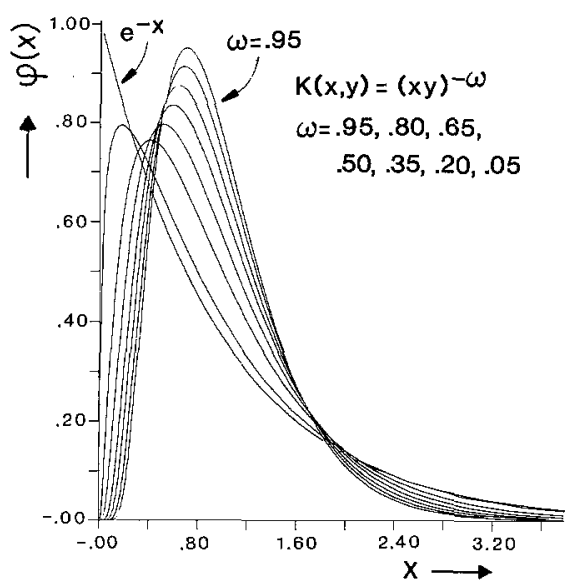

FIG. 1. Bell-shaped scaling function $\varphi_{\omega}(x)$ for the indicated class III kernel at $\omega=0.95(0.15) 0.05$, compared with $\varphi_{0}(x)=e^{-x}$ for $\omega=0$ (class II kernel). As $\omega \downarrow 0$ the right wing of $\varphi_{\omega}(x)$ approaches $\varphi_{0}(x)$ with typical crossover to class II behavior around $\omega=0.2$. However, $\varphi_{\omega}(x)$ as $x \downarrow 0$ shows always class III behavior. Results for $\omega=1$ and 0.5 agree with (7).

Journal of Colloid and Interface Science, Vol. 119, No. 2, October 1987 class II behavior at the "larger"- $x$ values. The lower boundary of this region shifts gradually toward zero as $\omega \downarrow 0$. However, the behavior of $\varphi_{\omega}(x)$ as $x \downarrow 0$ at fixed $\omega$ (no matter how small $\omega$ ) is always very different from that of $\varphi_{0}(x)=e^{-x}$ and always shows class III behavior. In fact, the calculated $\varphi(x)$ is by construction identical to the asymptotic form [13] for $x \leqslant \exp \left(z_{\mathrm{b}}\right)$, where $z_{\mathrm{b}}$ is defined below [19].

As an illustration of the accuracy of the method (no detailed error estimates have been made) we have calculated the moments $\bar{p}_{\gamma}$ of $\bar{\varphi}(\bar{x})$, as entering in Eq. [10] with $\alpha=\beta=-\omega$, and the RHS and the LHS $=(n=1) \bar{p}_{n}$ of this equation for $n=0,2,3, \cdots, 10$ and several values of $\omega$. The difference between both sides of Eq. [10] for $n \leqslant 8$ is less than $0.5 \%$. For larger- $n$ and large- $\omega$ values it increases till about $3 \%$.

The numerical results for $x \gg 1$ should approach the large- $x$ behavior $A x^{-2 \omega} e^{-a x}$, predicted in [11]. To test this we have plotted $\{\ln \varphi(x)+\lambda \ln x\}$ versus $x$ where $\lambda=-2 \omega$ is the degree of homogeneity, and made a leastsquares fit to $-a x+\ln A$ at the largest $x$ values considered. The former curves appear not to be completely straight, but right above Eq. [20] we have explained why numerical complications appear in the tail of the distribution. Table I compares the numerical computed value of $a / A$ with the analytic value $a / A=B(1+\omega$, $1+\omega)$ in Eq. [12]. The numerical value tends to be too high, but the difference is always less than $20 \%$. Table I also contains the values of $\bar{p}_{-\omega}$ that enter in the asymptotic form [13], the values of $\bar{p}_{0}$ and $\bar{p}_{1}$ used in rescaling $\bar{\varphi}$ to $\varphi$, and the number $N$ of abscissas used in the calculations.

Calculations for the same kernel $(x y)^{-\omega}$ with $\omega=0.5$ and 1.0 have been performed by Kolb (7). His values agree well with ours.

$$
\text { Kernel 2: } K(x, y)=x^{-\omega}+y^{-\omega}
$$

The second kernel [6] to be considered has exponents $\alpha=0$ and $\beta=-\omega$. Also in this case the integral equation [8] contains only a single unknown moment $\bar{p}_{0}$. The reason is that the 
TABLE I

Characteristics of Numerical Solution for $K(x, y)=2(x y)^{-\omega}$

\begin{tabular}{ccccccc}
\hline$\omega$ & $\bar{p}_{-\omega}$ & $\bar{p}_{0}$ & $\bar{p}_{1}$ & $N$ & $(a / A)_{\text {theor }}$ & $(a / A)_{\text {oum }}$ \\
\hline 0.05 & 0.1665 & 0.02773 & $1.255 \times 10^{-17}$ & 50 & 0.91 & 0.93 \\
0.20 & 0.2566 & 0.06588 & $1.064 \times 10^{-4}$ & 55 & 0.68 & 0.71 \\
0.35 & 0.3357 & 0.1128 & $6.854 \times 10^{-3}$ & 60 & 0.51 & 0.55 \\
0.50 & 0.4023 & 0.1619 & 0.03491 & 60 & 0.39 & 0.44 \\
0.65 & 0.4578 & 0.2096 & 0.08230 & 60 & 0.30 & 0.35 \\
0.80 & 0.5037 & 0.2538 & 0.1386 & 60 & 0.23 & 0.28 \\
0.95 & 0.5420 & 0.2938 & 0.1964 & 70 & 0.18 & 0.21 \\
\hline
\end{tabular}

first equation of [10] implies the equality $\bar{p}_{-\omega}$ $=1$ in the "numerical normalization" $(C=w$ $=1$, see above Eq. [17]). In Fig. 2 the calculated scaling function is used in the standard normalization. The comments about trends and accuracy of moment equations and ratio $a / A$ are essentially the same as those for Fig. 1 , with the exception of the case $\omega=0.05$, which is missing. The reason is that one is dealing with numbers that are too small (for $\omega=0.2, p_{10}=6.6 \times 10^{-91}$; for $\omega=0.05$, still smaller moments are involved). Table II compares again the numerically computed values of $a / A$ with the theoretical prediction $B(1,1$ $+\omega)=(1+\omega)^{-1}$ and lists the moments $\bar{p}_{0}$ entering in the $\varphi(x)$ equation, the moments $\bar{p}_{1}$, and the number $N$ of abscissas used.

$$
\text { Kernel 3: } K(x, y)=x^{\alpha} y^{-\omega}+y^{\alpha} x^{-\omega}
$$

In our last example we consider a fixed exponent $\alpha>0$ ( $\alpha=0.5$ and 0.9 have been chosen) and study the case $\omega \downarrow 0$. The limitng class II kernel is $K(x, y)=x^{\alpha}+y^{\alpha}$ with an unknown scaling function $\varphi(x)$. The small- $x$ behavior of the scaling function is $\varphi(x) \simeq B x^{-\tau}(x \downarrow 0)$ with $B$ undetermined. Analytical considerations only link the exponent $\tau$ to moments of $\varphi(x)$, i.e., $\tau=2-p_{\alpha} / w$. Again we study the crossover from typical class III to typical class II behavior as $\omega \downarrow 0$.

The numerical accuracy of our integration method for the examples in this subsection is considerably lower than that in the two preceding subsections. The main reason is as follows. Our method for evaluating the gain term is particularly suited for typical class III behavior (see Section 3). In this case, however, there occurs an extended intermediate range of $x$ values over which the behavior is completely different. Another difficulty is that the moment $\bar{p}_{-\omega}$ starts to diverge as $\omega \downarrow 0$, as can be seen from the inequality [16] combined with [14].

Also, the standard normalization [22] of the previous subsection can no longer be used in the limit as $\omega \downarrow 0$, because $\bar{p}_{0}$ starts to diverge for the same reason as $\bar{p}_{-\omega}$ does. An obvious alternative is the normalization based on the mass-average degree of polymerization by setting

$$
p_{1}=1 ; \quad p_{2}=1
$$

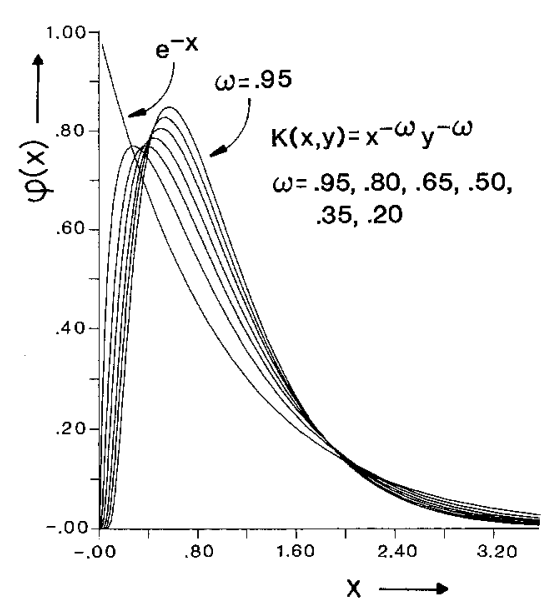

FIG. 2. Crossover behavior of $\varphi_{\omega}(x)$, similar to that in Fig. 1, from class III to class II as $\omega \downarrow 0$ for the kernel indicated, using the same normalization [22] as in Fig. 1. 
TABLE II

Characteristics of Numerical Solution for $K(x, y)=x^{-\omega}+y^{-\omega}$

\begin{tabular}{ccccccc}
\hline$\omega$ & $\bar{p}_{-\omega}$ & \multicolumn{1}{c}{$\bar{p}_{0}$} & $\bar{p}_{1}$ & $N$ & $(a / A)_{\text {troor }}$ & $(a / A)_{\text {num }}$ \\
\hline 0.20 & 1 & 0.01199 & $4.796 \times 10^{-12}$ & 45 & 0.83 & 0.87 \\
0.35 & 1 & 0.1002 & $2.175 \times 10^{-4}$ & 50 & 0.74 & 0.77 \\
0.50 & 1 & 0.2310 & 0.01869 & 55 & 0.67 & 0.69 \\
0.65 & 1 & 0.3586 & 0.1104 & 60 & 0.61 & 0.62 \\
0.80 & 1 & 0.4686 & 0.2676 & 60 & 0.56 & 0.57 \\
0.95 & 1 & 0.5594 & 0.4431 & 60 & 0.51 & 0.53 \\
\hline
\end{tabular}

(see Introduction). This can be achieved by rescaling, viz. $\varphi(x)=\eta \bar{\varphi}(x / \xi)$ with $\xi=\bar{p}_{1} / \bar{p}_{2}$ and $\eta=\bar{p}_{2}^{2} / \bar{p}_{1}^{3}$.

This normalization is also acceptable for class II kernels $(\omega \downarrow 0 ; \alpha \leqslant 1)$. Figures 3 and 4 show the numerical results for $\alpha=0.5$ and 0.9 in the standard normalization [23]. For $\alpha$ $=0.5$ the scaling function in Fig. 3 keeps increasing as $x$ decreases until $x_{0} \simeq 10^{-4}$ (typical class II behavior), where $\varphi(x)$ itself becomes very large. Its maximum increases with decreasing $\omega$, where the following set of $\omega$ values has been considered, $\omega=0.20,0.12,0.11,0.10$, $0.09,0.08$ (for larger- $\omega$ values $\varphi$ cannot be distinguished from the $x$ axis). Class III behavior

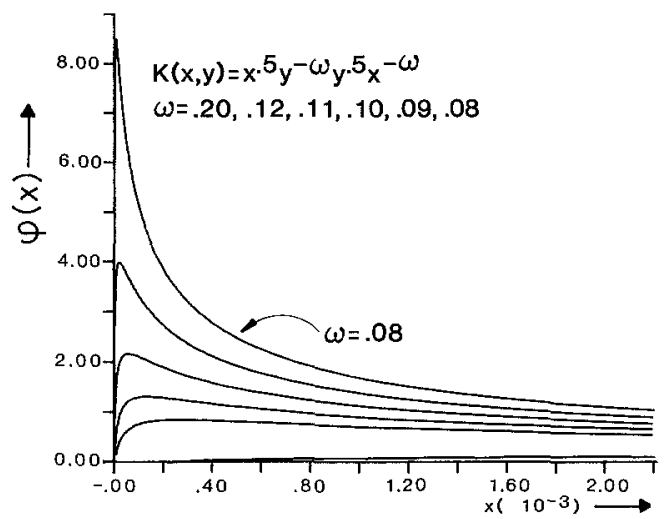

FIG. 3. For the kernel indicated, class III behavior of $\varphi_{\omega}(x)$ is confined to a tiny region $\left(\leqslant 5 \times 10^{-4}\right)$ around the origin, shrinking with decreasing $\omega$. The large increase in $\varphi$ values as $\omega \downarrow 0$ supposedly indicates the approach of $\varphi_{\omega}(x)$ to typical algebraic class II behavior, $\varphi_{0}(x) \sim x^{-\tau}$ as $x \downarrow 0$ for $K=\sqrt{x}+\sqrt{y}$ with $\tau \simeq 1.12$ calcualted from [24]. The $\varphi$ and $x$ units, determined by the normalization [23], are different from those in Figs. 1 and 2. can only be seen in a tiny region of $x$ values with $x \leqslant 10^{-4}$. The curves in Fig. 4 for $\alpha=0.9$ and $\omega=0.3,0.2$, and 0.15 (for larger- $\omega$ values $\varphi$ cannot be distinguished from the $x$ axis) show more or less the same features. At still smaller- $\omega$ values our numerical algorithm did not yield a solution, because no fixed point could be found, due to the inaccuracy of the method used for the evaluation of the gain term.

We further compared the RHS and LHS of the moment equation [10] with $n \leqslant 10$. The difference between both sides depends on $n$, and is smaller than $1 \%$ for small $n$; as $n$ grows, it amounts to several percent (for both $\alpha=0.5$ and $\alpha=0.9$ ). It was also observed that the numerical results for $a / A$ no longer corresponded to the analytical prediction [12]. The

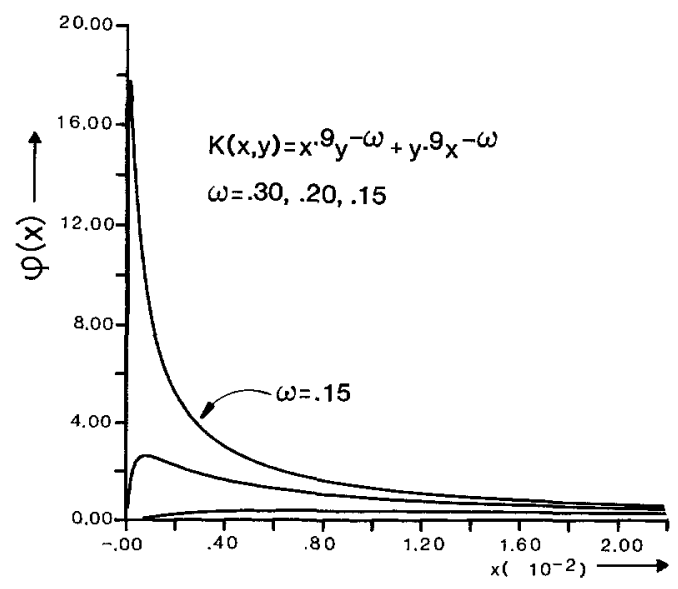

FIG. 4. For the kernel indicated $\varphi_{\omega}(x)$ shows trends similar to those in Fig. 3. 
TABLE III

Characteristics of Numerical Solution for $K(x, y)=x^{\alpha} y^{-\omega}+y^{\alpha} x^{-\omega}(\alpha=0.5)$

\begin{tabular}{lllllll}
\hline$\omega$ & $\bar{p}_{\alpha}$ & \multicolumn{1}{c}{$\bar{p}_{-\omega}$} & \multicolumn{1}{c}{$\bar{p}_{1}$} & \multicolumn{1}{c}{$\bar{p}_{2}$} & \multicolumn{1}{c}{$N$} & $\tau(\omega)$ \\
\hline 0.5 & 1.388 & 0.5678 & 2.933 & 19.91 & 60 & 1.209 \\
0.4 & 1.104 & 0.6746 & 2.014 & 10.59 & 58 & 1.205 \\
0.3 & 0.8245 & 1.015 & 1.037 & 2.708 & 57 & 1.194 \\
0.2 & 0.5498 & 3.140 & 0.2473 & 0.09156 & 110 & 1.174 \\
0.12 & 0.3644 & 41.22 & 0.01416 & $4.270 \times 10^{-5}$ & 120 & 1.158 \\
0.11 & 0.3433 & 76.73 & 0.007342 & $6.827 \times 10^{-6}$ & 125 & 1.156 \\
0.10 & 0.3228 & 163.5 & 0.003339 & $7.353 \times 10^{-7}$ & 130 & 1.153 \\
0.09 & 0.3028 & 417.8 & 0.001267 & $4.621 \times 10^{-8}$ & 130 & 1.149 \\
0.08 & 0.2833 & 1373 & 0.0003748 & $1.385 \times 10^{-9}$ & 135 & 1.146 \\
\hline
\end{tabular}

ratio was about a factor of 1.3 too small for the case $\alpha=0.5$, and a factor of 3 for the case $\alpha=0.9$. This is probably caused by the numerical inaccuracies already mentioned.

To obtain some quantitative information on the class II kernels, about which hardly anything is known $(5,10)$, we try to calculate the $\tau$ exponent defined in [14] for the class II kernel $K(x, y)=x^{\alpha}+y^{\alpha}$. This will be done by using the class III results for $\varphi_{\omega}$ at small $\omega$, together with the analytic result [15] and extrapolating it to $\omega=0$. Since $\varphi_{0}(x)$ is not known, a direct evaluation of [15] is not possible.

The extrapolation is carried out by introducing the quantity

$$
\tau(\omega)=2-p_{\alpha-\omega} / w=2-\bar{p}_{\alpha-\omega}
$$

for the class III kernel, and studying its limiting behavior as $\omega \downarrow 0$. The introduced quantity $\tau(\omega)$ is independent of the rescaling freedom $\eta$ and $\xi$, as is the $\tau$ exponent [15] for class II.
For its calculation, evaluation of the integral $\bar{p}_{\alpha-\omega}$ is required.

The approach of $\varphi_{\omega}(x)$ to $\varphi_{0}(x)$ for small values of $x$ may be illustrated as follows. We take the numerically determined fixed point value $\bar{p}_{\alpha}(\omega)$ for the coefficient entering in the $\varphi$ equation for $K=x^{\alpha} y^{-\omega}+y^{\alpha} x^{-\omega}$. Hence, the small $x$ behavior

$$
\bar{\varphi}_{\omega}(\bar{x}) \simeq(\bar{x})^{-2} \exp \left[-\bar{p}_{\alpha}(\omega)(\bar{x})^{-\omega} / \omega\right]
$$

is known explicitly. Next we rescale to the standard normalization [23] (appropriate for $\omega \rightarrow 0$ ), with $\xi$ and $\eta$ given below [23], using the numerically determined values of $\bar{p}_{1}$ and $\bar{p}_{2}$. The small- $x$ behavior of the rescaled class III function becomes

$$
\begin{aligned}
\varphi_{\omega}(x) & \simeq \eta \xi^{2} x^{-2} \exp \left[-\bar{p}_{\alpha}(\omega) \xi^{\omega} x^{-\omega} / \omega\right] \\
& =B_{\omega} x^{-2} \exp \left[\left(p_{\alpha}(\omega) / \omega\right)\left(1-x^{-\omega}\right) / \omega\right]
\end{aligned}
$$

with $p_{\alpha}(\omega) / w=\bar{p}_{\alpha}(\omega) \xi^{\omega}$. In the limit as $\omega \downarrow 0$

\begin{tabular}{|c|c|c|c|c|c|c|}
\hline$\omega$ & $\bar{p}_{\alpha}$ & $\tilde{p}_{-\omega}$ & $\bar{p}_{1}$ & $\bar{p}_{2}$ & $N$ & $\tau(\omega)$ \\
\hline 0.5 & 1.403 & 0.2418 & 1.734 & 22.50 & 125 & 1.434 \\
\hline 0.4 & 1.081 & 0.3262 & 1.308 & 14.41 & 128 & 1.431 \\
\hline 0.3 & 0.7283 & 0.7949 & 0.8138 & 4.345 & 130 & 1.429 \\
\hline 0.2 & 0.3720 & 16.23 & 0.3080 & 0.09127 & 140 & 1.425 \\
\hline 0.15 & 0.2173 & 1087 & 0.1177 & 0.0005000 & 149 & 1.420 \\
\hline
\end{tabular}
Eq. [25] approaches the class II result at small

TABLE IV

Characteristics of Numerical Solution for $K(x, y)=x^{\alpha} y^{-\omega}+y^{\alpha} x^{-\omega}(\alpha=0.9)$ 

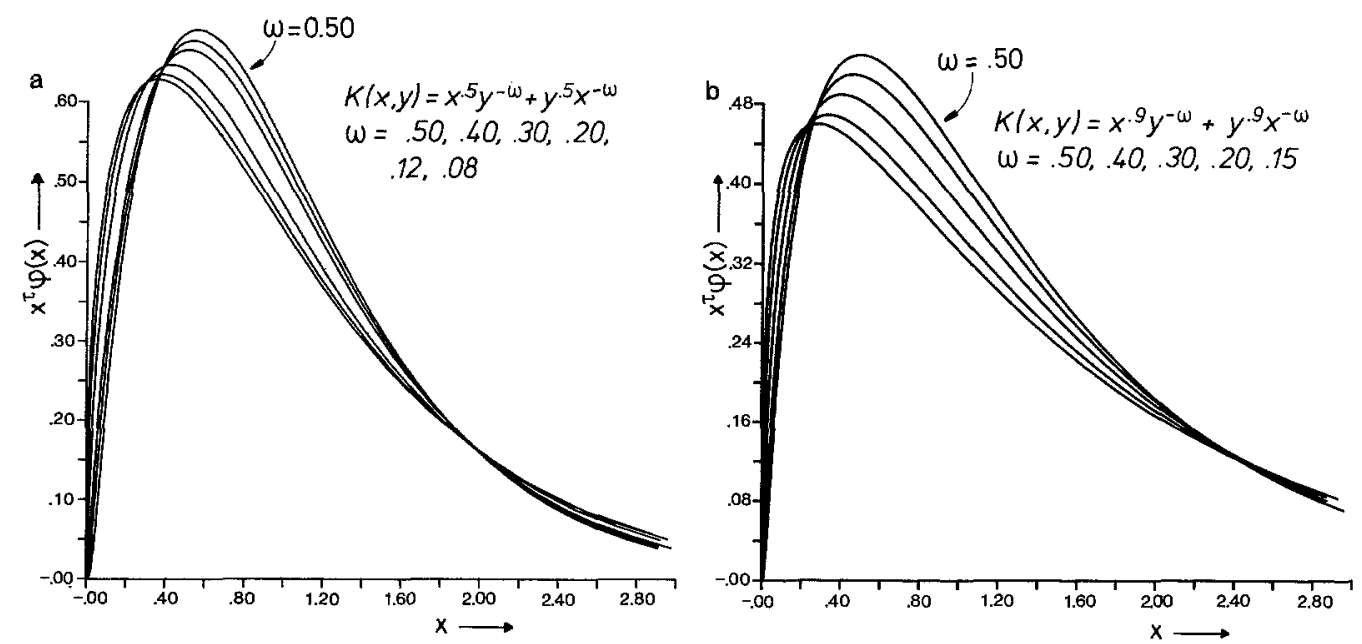

FiG. 5. (a and b) The same data as in Figs. 3 and 4, plotted as $x^{\tau} \varphi(x)$ (with $\tau=1.12$ (a), 1.41 (b)) over a much larger $x$ interval, showing a smooth variation of this quantity with $\omega$.

$x$, given by $\varphi_{\omega 10}(x)=B x^{-\tau}$ with $\tau=\tau(\omega \downarrow 0)$, where $\tau(\omega)$ is defined by [24].

The values for the quantities $\tau(\omega)$ in [24] at $\alpha=0.5$ and 0.9 are listed in Tables III and IV, respectively. From Table III we obtain the estimate $\tau \simeq 1.12$ as $\omega \downarrow 0$ for the $\tau$ exponent of the class II kernel $K(x, y)=\sqrt{x}+\sqrt{y}$. This result lies well between the bounds [16] and also between the better bounds $1<\tau<1.28$, derived in (10). Table IV shows similar results for $\tau(\omega)$ at $\alpha=0.9$. For this case the estimate $\tau(\omega \downarrow 0)=1.41$ is obtianed, lying within the predicted bounds $1.12<\tau<1.46$ (see Ref. (10)).

Finally we have plotted in Figs. 5a and 5b the graphs for $x^{\tau} \varphi(x)$ in the standard normalization [23] for the class III kernel $K(x, y)$ $=x^{\alpha} y^{-\omega}+y^{\alpha} x^{-\omega}$ with $\alpha=0.5$ and $\alpha=0.9$, respectively. The graphs show the interesting feature that $x^{\tau} \varphi(x)$ depends only weakly on $\omega$, as opposed to $\varphi(x)$ itself, as shown in Figs. 3 and 4.

\section{CONCLUSION}

We have numerically studied the scaling function of "self-preserving spectrum" (1) for aggregation processes described by Smoluchowski's coagulation equation. This has been done for the factorizable class III kernels $(x y)^{-\omega}, x^{-\omega}+y^{-\omega}$, and $x^{\alpha} y^{-\omega}+y^{\alpha} x^{-\omega}(\alpha=0.5$ and 0.9 ) for several values of $\omega$.

The analytic predictions obtained from scaling theory $(2,6)$ have been confirmed with satisfactory accuracy and have been supplemented with many quantitative data. Special emphasis was put on the crossover behavior of $\varphi(x)$ from class III (bell-shaped $\varphi$ curve) to class II behavior (algebraic small- $x$ behavior, $x^{-\tau}$ ). We have further shown how the $\tau$ exponent for the class II kernels $K(x, y)=x^{\alpha}$ $+y^{\alpha}$ can be calculated, and obtained $\tau=1.12$ at $\alpha=0.5$, and $\tau=1.41$ at $\alpha=0.9$.

The accuracy of our numerical algorithm can still be improved, but we have not pursued that line of research.

\section{ACKNOWLEDGMENTS}

It is our pleasure to thank $P$. van Dongen for many clarifying discussions. One of us (M.H.E.) thanks IPST at the University of Maryland for its hospitality and financial support during the summer and fall of 1986, when this research was completed.

\section{REFERENCES}

1. Friedlander, S. K., "Smoke, Dust and Haze." Wiley, New York, 1977.

2. Viscek, T., and Family, F., Phys. Rev. Lett. 52, 1669 (1984); Meakin, P., Viscek, T., and Family, F., 
Phys. Rev. B 31, 564 (1985); Mulholland, G. W., and Mountain, R. D., J. Chem. Phys. 84, 4109 (1986).

3. Ziff, R. M., McGrady, E. D., and Meakin, P., J. Chem. Phys. 82, 5269 (1985); Kang, K., Redner, S., Meakin, P., and Leyvraz, F., Phys. Rev. A 33, 1171 (1986).

4. See, e.g., the review by Herrmann, H. J., Phys. Rep. 136, 154 (1986).

5. Samson, R. J., Mulholland, G. W., and Gentry, J. W., preprint, Oct. 1986.

6. van Dongen, P. G. J., and Ernst, M. H., Phys. Rev. Lett. 54, 1396 (1984).

7. Kolb, M., Phys. Rev. Lett. 53, 1653 (1984).

8. Ernst, M. H., Hendriks, E. M., and Leyvraz, F., $J$. Phys. A 17, 2137 (1984); Ernst, M. H., in "Fractals in Physics" (L. Pietronero and E. Tosatti Eds.). Elsevier, Amsterdam, 1986.

9. Lushnikov, A. A., and Piskunov, V. N., Dokl. Akad. Nauk. SSSR 231, 1166 (1976).

10. van Dongen, P. G. J., and Ernst, M. H., Phys. Rev. $A$ 32, 670 (1985).

11. Swift, D. L., and Friedlander, S. K., J. Colloid Interface Sci. 19, 621 (1964).

12. Collatz, L., "Numerische Behandlungvon Differentialgleichungen," p. 79. Springer, Berlin 1955.

13. Abramowitz, M., and Stegun, I. A., "Handbook of Mathematical Functions," p. 923. Dover, New York, 1972.

14. Gill, P. E., and Murray, W., Minimization Subject to Bounds on the Variables. National Physical Laboratory Report NAC 72, 1976. 